\title{
Quiz of the Month
}

\section{Questions}

Submitted by Eben I. Feinstein, MD, Associate Editor. These questions are based upon information contained in the Editorial Reviews, Renal Physiology Seminars and Nephrology Consultant sections of the American Journal of Nephrology, vol. 9 (1989). From the statements in each question, select the one that is not true. Questions 1 Concerning lysophospholipids (LPC): a Their natriuretic action is due to inhibition of membrane Na-K-ATPase. b LPC causes erythrocyte lysis by increasing membrane permeability to cations. c LPC may play a role in the ventricular arrhythmias seen after myocardial infarction. $\mathrm{d}$ Volume expansion causes an increase in the plasma concentration of LPC. 2 In the diagnosis of pYmicroglobulin (pYM) amyloidosis. a High serum levels of $\mathrm{p}$ \&gt; $\mathrm{M}$ strongly suggest the presence of pYM amyloidosis. b Lytic lesions of pVM amyloidosis in the spine are usually not surrounded by sclerosis. c Radionuclide bone scans usually give false-negative results. d Electron microscopy of biopsy specimens from affected areas show curvilinear, randomly arranged fibrils of a diameter of 8-10 $\mathrm{nm}$.

3 The kidneys of patients with HIV-associated nephropathy show:

a Microcystic dilatation of the tubules.

b HIV core or HIV envelope protein antigens in the glomerular deposits of patients with an immune complex glomerulonephritis. c HIV proviral DNA in tubular and glomerular epithelial cells. 